\title{
Osteogenesis imperfecta: from bench to bedside
}

\author{
Roland Kocijan
}

Published online: 14 July 2015

(C) Springer-Verlag Wien 2015

Osteogenesis imperfecta (OI) is a group of rare, inherited disorders with genetic and clinical diversities. With a prevalence of 1:10,000-1:20,000, OI is considered as an "orphan disease." OI is caused by quantitative or qualitative defects in collagen type 1 or by proteins interacting with collagen type 1 . Changes in the amount or in the structure of collagen type 1 result in high bone fragility. Therefore, $\mathrm{OI}$ is also known as brittle bone disease.

In severe cases, fracture occurrence starts during the intrauterine or perinatal period leading to multiple fractures of hollow bones and ribs, which results in respiratory insufficiency and death. In contrast, mild OI leads to fewer fractures and in this respect mimics juvenile osteoporosis. Deformity of the skeleton and extraskeletal features such as dentinogenesis imperfecta and blue sclerae are typical signs of OI. Based on these characteristics, Professor David Sillence introduced his classification in 1979. He divided patients into four subgroups, suggesting dominant and recessive genotypes responsible for OI. The original Sillence classification has been expanded to include 11 different types of OI based on genetic alterations [1]. In respect to the clinical classification of OI, Sillence is (still) golden.

In this special issue, a detailed overview of the historical and the most recently published classifications of OI is presented. Besides the more common autosomal dominant forms, rare and recessive OI types are also described (Fratzl-Zelman et al.).

In patients with OI, bone mineralization is abnormally high, whereas bone mineral density is usually decreased. Consequently, bones loose elasticity and are more likely to fracture. This phenomenon of high bone mineralization in patients with $\mathrm{OI}$ is reported by Fratzl-Zelman et al.

As bone turnover is high in severe OI and fracture occurrence starts in childhood, a specific therapy is recommended in the first year of life. Bisphosphonates are considered as reliable agents in the treatment of juvenile and adult OI. The Receptor Activator of NF-KB Ligand (RANKL) inhibition by Denosumab and the osteoanabolic agent teriparatide (TPTD) are promising new therapeutic options (Semler et al.).

The coexistence of hypogonadotropic hypogonadism and OI results in heightened deterioration of bone microstructure, multiple fractures, and a delay in fracture healing, as reported by Plachel et al. in a patient with clinical OI type III. To increase trabecular bone volume and to accelerate fracture healing, a TPTD treatment was initiated.

In this special issue on OI, we build a bridge from bench to bedside: from genetic and clinical classification, diagnostic tools including the assessment of high bone matrix mineralization and radiographic characteristics up to established and new treatment modalities.

\section{Conflict of interest}

The author declares no conflict of interest.

\section{Reference}

1. Forlino A, Cabral WA, Barnes AM, Marini JC. New perspectives on osteogenesis imperfecta. Nat Rev Endocrinol. 2011;7:540-57.

R. Kocijan, MD $(\bowtie)$

St. Vincent Hospital Vienna, Medical Department II,

Academic Teaching Hospital of the Medical University Vienna,

Stumpergasse 13,

1060 Vienna, Austria

e-mail: roland.kocijan@bhs.at 